\title{
Investigation of Carbon Deposition on Ni/Gd Doped Ceria Reforming Catalysts for Solid Oxide Fuel Cells
}

\author{
Q. Liu and P.A. Crozier
}

School for Engineering of Matter, Transport and Energy, Arizona State University, Tempe, AZ 852876106

Intermediate temperature solid oxide fuel cells (IT-SOFC) are promising electrochemical conversion devices that produce electricity by oxidizing chemical fuels. One of the most attractive advantages of ITSOFCs is that, in principle, various types of fuels (hydrogen, hydrocarbons...) can be utilized. One desired approach in practice is to divide the anode into two different functionality regions: (1) an internal reforming layer that converts hydrocarbon fuels to hydrogen; (2) an electrocatalytically active layer that oxidizes the hydrogen and collects electrons. Our research focusses on Ni/Gd co-doped ceria anode material in which Ni serves as both the electron collector and the reforming catalyst. However, when carbonaceous fuels are employed, under certain conditions carbon can deposit onto Ni surfaces which can significantly degrade or even destroy the anode. While Ni catalyzes carbon growth, ceria is known to be an oxidation catalyst and thus suitably structured combinations of ceria and Ni may result in suppressed carbon formation. To explore this hypothesis it is necessary to conduct a fundamental investigation of the carbon deposition mechanism on $\mathrm{Ni} / \mathrm{Gd}$ co-doped ceria catalyst.

The reforming nanopowders consist of $20 \mathrm{at} \% \mathrm{Ni}, 15$ at $\% \mathrm{Gd}$ doped ceria were synthesized using a spray drying technique [1]. The powders were then calcined at $500^{\circ} \mathrm{C}$ and $700^{\circ} \mathrm{C}$ for 4 hours to form fresh reforming catalyst. Catalytic reforming characterization was investigated for partial oxidation of methane (POM) by heating the fresh catalyst in flowing methane and oxygen gases in an ISRI RIG-150 microreactor and the exit gases were analyzed using a gas chromatography (Varian GC-450). Methane conversion for POM was observed to be close to the thermodynamic equilibrium value at $650^{\circ} \mathrm{C}$. Then the oxygen flow was reduced to deliberately deposit carbon onto the catalyst as a result of the methane decomposition reaction. A JEOL $2010 \mathrm{~F}$ was employed to obtain high resolution images and energy dispersive x-ray spectroscopy (EDS) of both the fresh and carbon deposited catalyst to determine the structure and composition of the material and the form and location of the carbon layer.

The morphology of the fresh catalyst is shown in Fig.1. Diffractogram analysis of this image shows spots from both Gd doped ceria (GDC) grains and $\mathrm{NiO}$ grain. This reveals that some of the Ni has separated from the ceria grains during preparation. Fig. 2 shows the reactor data from the carbon deposition experiment in which methane conversion decreased as the oxygen flow was reduced. Typical TEM images from the used catalyst are displayed in Fig.3 which proves that a significant quantity of carbon has been deposited on both Ni metal and GDC. The observed carbon morphology and lattice spacings reveal that it is in the form of graphite. EDS spectra (Fig.4) show high $\mathrm{C}$ and Ni peaks and low $\mathrm{Ce}$ peaks in the used catalyst which suggests that graphite tends to form in Ni rich regions. In this preliminary experiment, the oxygen flow was decreased almost to zero and an excessive amount of carbon has been deposited. Therefore it is not possible to determine the initial nucleation sites for graphite formation. Moreover, during the reaction, $\mathrm{CeO}_{2}$ may be reduced to $\mathrm{Ce}_{2} \mathrm{O}_{3}$ which would not be able to oxidize the carbon. Additional experiments will be performed to deposit smaller quantities of carbon under conditions that are more typical for fuel cell anodes. In situ experiments will also be carried out to determine the oxidation state of Ce during graphite formation [2]. 


\section{References}

[1] V. Sharma et al., Chem. Phys. Lett., 495 (2010) 280.

[2] The financial support from Arizona State University and the use of ETEM at the John M. Cowley Center for High Resolution Electron Microscopy at Arizona State University is gratefully acknowledged.

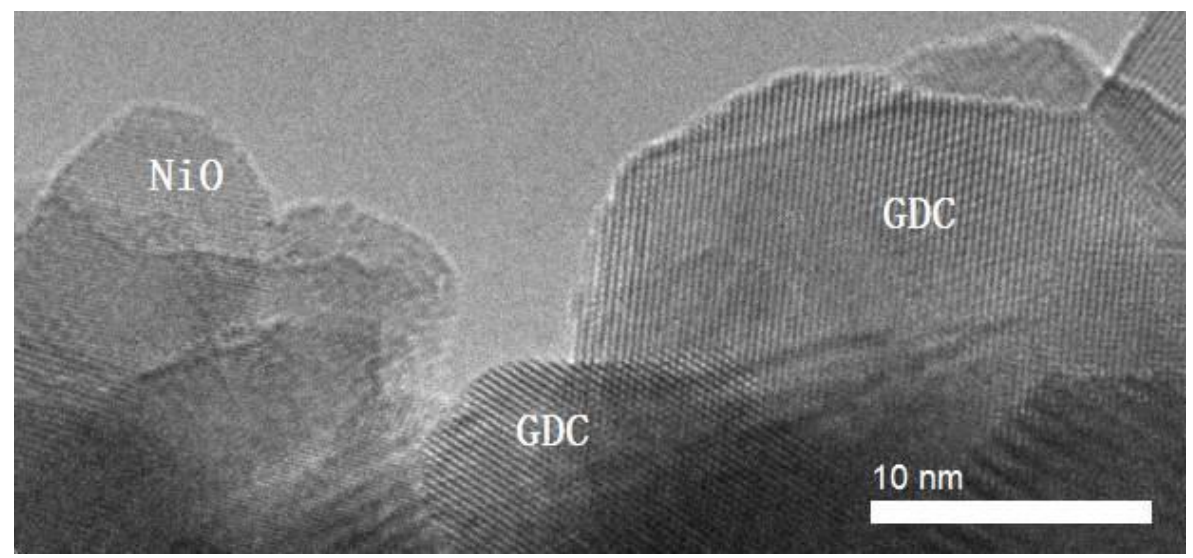

Figure 1. TEM image of fresh catalyst showing $\mathrm{NiO}$ and GDC grains.

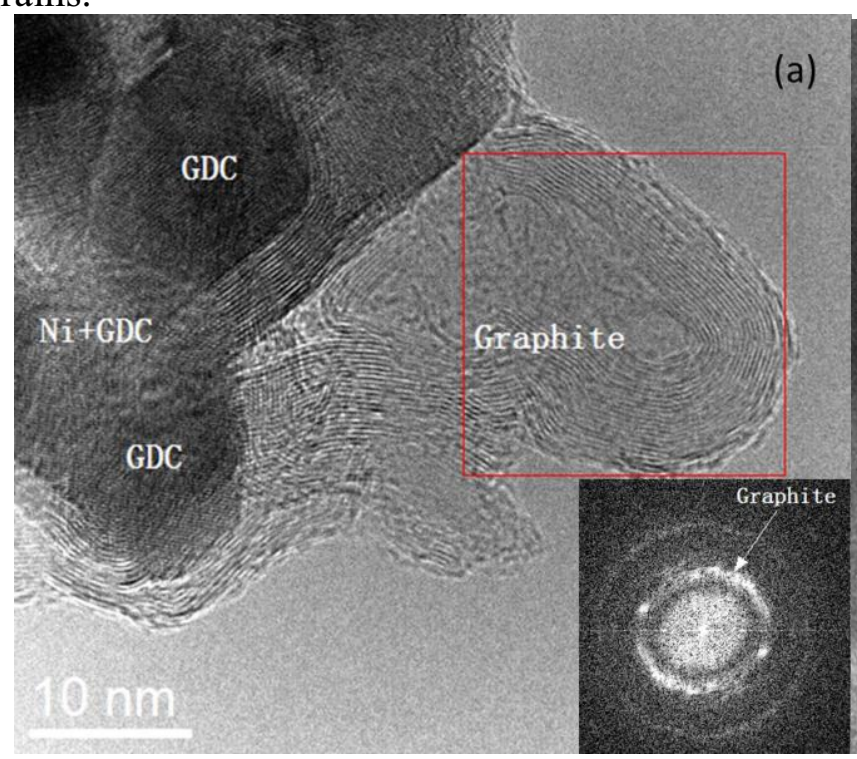

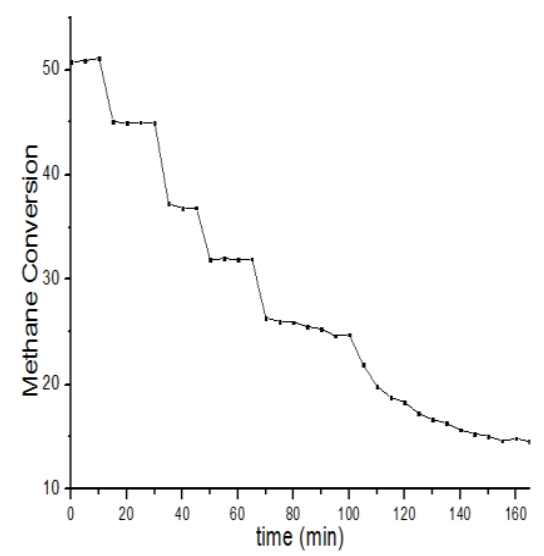

Figure 2. Methane conversion for POM decreasing with time.

Figure 3. Typical TEM images of graphite deposited on $\mathrm{Ni}$ metal and adjacent $\mathrm{CeO}_{2}$ grains.
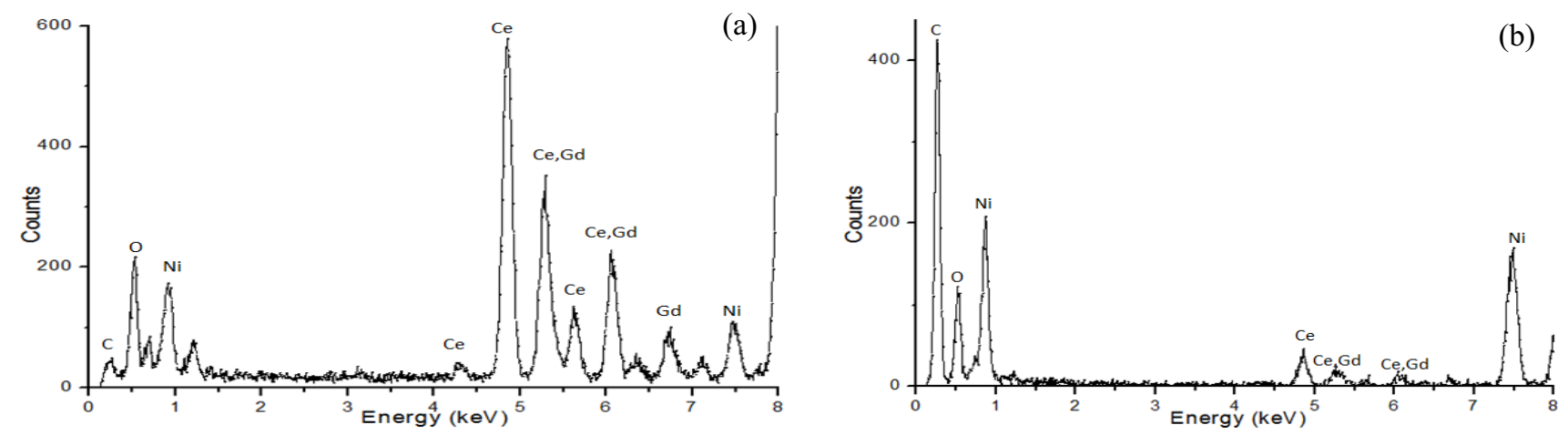

Figure 4. EDS spectra of (a) fresh catalyst. (b) carbon deposited catalyst. 